\title{
Optimal startup operation of simulated moving bed chromatographic processes
}

\author{
Suzhou Li ${ }^{*}$ Yoshiaki Kawajiri ${ }^{* *}$ Jörg Raisch ${ }^{* * * *}$ \\ Andreas Seidel-Morgenstern ${ }^{*, * * *, 1}$ \\ * Max-Planck-Institut für Dynamik komplexer technischer Systeme, \\ Sandtorstraße 1, D-39106 Magdeburg, Germany \\ ** School of Chemical 83 Biomolecular Engineering, Georgia Institute \\ of Technology, 311 Ferst Drive, Atlanta, GA 30332, USA \\ *** Fachgebiet Regelungssysteme, Technische Universität Berlin, \\ Einsteinufer 17, D-10587 Berlin, Germany \\ **** Lehrstuhl für Chemische Verfahrenstechnik, Otto-von-Guericke \\ Universität, Universitätsplatz 2, D-39106 Magdeburg, Germany
}

\begin{abstract}
SMB represents one of the widely established periodic adsorption processes and its periodic and nonlinear dynamics presents a significant challenge to the formulation and solution of the optimal startup issue. A multistage startup concept allowing to adjust operating conditions stage-wise is proposed. The startup problem is then formulated aiming at driving the system towards the reference cyclic steady state (CSS) in an optimum manner. A tailored decomposition algorithm is developed to tackle the resulting optimization problem and guarantee numerical tractability. The feasibility of the solution approach is demonstrated on a binary separation with nonlinear competitive isotherms. It is found that the new startup policy dramatically reduces transient time and desorbent consumption. The startup performance in terms of product concentration and purity is also evaluated quantitatively.
\end{abstract}

Keywords: simulated moving bed chromatography, startup, transient behavior, optimization

\section{INTRODUCTION}

Simulated moving bed (SMB) chromatography developed by UOP in the 1960s, has emerged as a continuous and efficient separation technology. Compared to the conventional elution chromatography, it enables high throughput and low solvent consumption and thus has been successfully applied in petrochemical, sugar and fine chemical industries. In the last decade, there has been a remarkably increased interest in extending SMB to pharmaceutical applications.

The SMB process as shown in Fig. 1 is designed as a practical implementation of the true moving bed (TMB) operation. The process consists of multiple identical chromatographic columns connected in series to form a closed loop. The inlet and the outlet ports divide the unit into four distinct zones with specific roles during the separation of a binary mixture of A and B. To mimic the countercurrent movement in TMB, the positions of the input and withdrawal ports are periodically shifted by one column in the direction of the mobile phase flow at a predetermined switching period. Because of the periodic port movement along the circularly arranged columns, following startup the process achieves the cyclic steady state (CSS) characterized by identical transient concentrations inside the columns and at both outlets within each switching period.

${ }^{1}$ Corresponding author. Tel.: +49-391-67-18643; fax: +49-391-6712028. E-mail: anseidel@vst.uni-magdeburg.de.

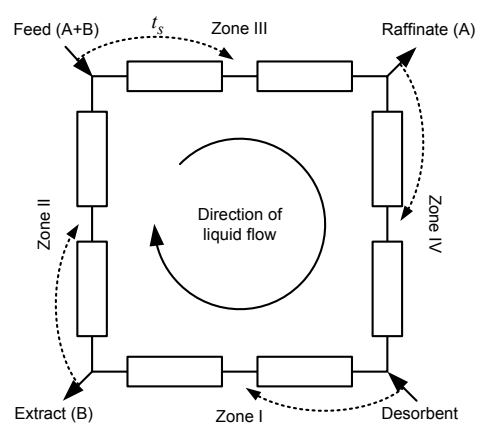

Fig. 1. Scheme of a four-zone simulated moving bed unit Since startup period is typically unproductive and off-spec products are produced (e.g., low concentration, purity, etc.), the CSS is used for production. For given separations, it is always desirable to reduce startup time and establish desired CSS as quickly as possible. In particular, for industrial SMB applications of large volume where emergency shutdown might occur, a fast startup operation allows to resume normal production in a short time and reduce non-productive duration. In the case of smallscale separation campaigns, the same SMB unit is used repeatedly to process small batches of different mixtures so that frequent feed changeovers and startups are needed. Efficient startup strategies become particularly critical and can significantly shorten changeover times for different products. However, in the SMB community most of the design and optimization methods focus on the cyclic steady state. The conventional startup procedure where operating 
conditions determined for CSS are directly set to the process is often applied. Only very few attempts have been made to explicitly account for startup performance. Lim and Ching (1996) proposed a pre-loading step and theoretically showed that such an additional step can shorten the startup period for their chiral separation. Xie et al. (2003) further enhanced this approach by developing a detailed design procedure of pre-loading and pre-elution for insulin purification. The new regime allows a significant reduction of the startup time for their tandem SMB system. To the best of our knowledge, however, no systematic studies of the optimal startup of SMBs have been reported so far in the open literature.

In this paper, a model-based multistage startup strategy is suggested for SMBs. The optimal startup problem is formulated which aims to minimize the temporal integration of the transient concentration deviation from the target CSS trajectory. Due to its numerical intractability, the overall startup optimization problem is decomposed into a sequence of subproblems that can be efficiently solved stage by stage. A lab-scale separation is employed to illustrate the feasibility of the decomposition approach and evaluate the potential of the proposed startup policy. The achievable transient performance is compared to that of the conventional startup scheme.

\section{MODELLING SMB STARTUP}

In order to quantitatively describe the transient behavior, an accurate mathematical model capable of characterizing the continuous chromatographic separation and periodical port switching is needed. Such an model can be assembled from the node balance equations and the dynamic models of single chromatographic columns. From the mass balances around the inlet and outlet nodes, one set of node equations can be identified:

Desorbent node:

Extract node:

$$
Q_{I V}+Q_{D}=Q_{I}, C_{i, I V}^{\text {out }} Q_{I V}=C_{i, I}^{i n} Q_{I}
$$

Feed node:

$$
Q_{I}-Q_{E}=Q_{I I}, C_{i, I}^{o u t}=C_{i, I I}^{i n}=C_{i}^{E}
$$

$$
Q_{I I}+Q_{F}=Q_{I I I}, C_{i, I I}^{\text {out }} Q_{I I}+C_{i}^{F} Q_{F}=C_{i, I I I}^{i n} Q_{I I I}
$$

Raffinate node:

$$
Q_{I I I}-Q_{R}=Q_{I V}, C_{i, I I I}^{\text {out }}=C_{i, I V}^{i n}=C_{i}^{R}
$$

where $Q_{j}(j=I, I I, I I I, I V)$ are the four internal flowrates, $Q_{D}$ the desorbent flow-rate, $Q_{E}$ the extract flowrate, $Q_{F}$ the feed flow-rate, $Q_{R}$ the raffinate flow-rate, $C_{i, j}^{\text {out }}$ and $C_{i, j}^{i n}$ the liquid concentrations of component $i$ leaving or entering zone $j, C_{i}^{E}$ and $C_{i}^{R}$ the concentrations of component $i$ at the extract and the raffinate outlet and $C_{i}^{F}$ the feed concentration of component $i, i=A, B$.

The well established equilibrium dispersive model (Guiochon et al. (2006)) was used to calculate the transient concentrations inside the columns. In this model the differential mass balance of component $i$ in each column can be written as:

$$
\frac{\partial C_{i}}{\partial t}+\frac{1-\epsilon}{\epsilon} \frac{\partial q_{i}}{\partial t}+v \frac{\partial C_{i}}{\partial z}=D_{a p, i} \frac{\partial^{2} C_{i}}{\partial z^{2}}, i=A, B
$$

where $C_{i}$ and $q_{i}$ are the concentrations of component $i$ in the liquid and the solid phase, respectively, $v$ the interstitial velocity of the liquid phase in the column, $z$ the spatial coordinate, $t$ the time, $\epsilon$ the total column porosity. The model assumes a local equilibrium between the solid and the liquid phase. Axial dispersion and mass transfer resistances which contribute to band broadening are lumped into an apparent dispersion coefficient $D_{a p, i}$. For simplicity, the same coefficient determined by (6) was used for both components:

$$
D_{a p, i}=\frac{v L}{2 N T P}
$$

where $L$ is the column length and $N T P$ the number of theoretical plates per column.

It is assumed that the separation campaign is started with clean columns, thus yielding the following initial conditions for each column:

$$
\left.C_{i}(t, z)\right|_{t=0}=0
$$

The boundary conditions at the inlet and outlet of the columns are specified by the Danckwerts relations:

$$
\begin{gathered}
\left.D_{a p, i} \frac{\partial C_{i}}{\partial z}\right|_{z=0}-v\left(\left.C_{i}\right|_{z=0}-C_{i}^{i n}\right)=0, \\
\left.D_{a p, i} \frac{\partial C_{i}}{\partial z}\right|_{z=L}=0
\end{gathered}
$$

Additionally, the adsorption equilibrium of the two components was described by the nonlinear isotherm of the competitive Langmuir-type:

$$
q_{i}\left(C_{A}, C_{B}\right)=\frac{H_{i} C_{i}}{1+K_{A} C_{A}+K_{B} C_{B}}, i=A, B
$$

with $H_{i}$ being the Henry constants and $K_{i}$ thermodynamic coefficients.

The overall system of coupled partial differential equations (PDEs) was discretized by the orthogonal collocation on finite elements (OCFE) approach, and the resulting differential algebraic equations (DAEs) were integrated using the DASPK3.1 package (Li and Petzold (1999)).

\section{CSS OPERATION AND CONVENTIONAL STARTUP}

The development of reliable design and operation procedures for SMBs has received considerable attention in the last decades. Representatives include the standing wave analysis suggested by Ma and Wang (1997) and the "triangle theory" approach proposed by Mazzotti et al. (1997). These short-cut design methodologies circumvent drawbacks of conventional trial-and-error methods, thus providing a valuable guide for the development of new applications of the SMB technology. However, both procedures are based on the equivalent TMB model and the "triangle theory" neglects the effect of axial dispersion and mass transfer resistances. To overcome these limitations, the model-based mathematical optimization methods have been developed (Dünnebier et al. (2000), Kawajiri and Biegler (2006), Toumi et al. (2007)). They consider a rigorous SMB model, involve a detailed cost function and use efficient Newton-based solution strategies. Nevertheless, both classes of methods reviewed aim at determining the operating conditions fulfilling the separation specifications only at the cyclic steady state, and they do not account for the transient behavior. Here, this set of conditions is referred to as the CSS conditions represented by vector 
$u^{*}$. The desired (or nominal) axial concentration profile established at CSS is denoted by vector $C_{a x}^{*}(t)$. It is assumed that $u^{*}$ and $C_{a x}^{*}(t)$ are known a priori throughout the paper.

In the conventional startup operation, SMBs with clean columns are started with the CSS conditions and the desired cyclic steady state can be reached typically after tens or hundreds of cycles. Simply using the CSS conditions as the transient operating policy can not guarantee a fast startup and more efficient regimes need to be developed.

\section{OPTIMAL STARTUP OPERATION OF SMB}

Due to the nonlinear and periodical nature inherent in SMB dynamics, formulating an optimal startup problem for such periodic adsorption process remains a challenge. Well-developed direct solution strategies, e.g., single or multiple-shooting methods and simultaneous approaches, which have been proven to be highly efficient for continuous processes and CSS optimization of SMB (Dünnebier et al. (2000), Kawajiri and Biegler (2006), Toumi et al. (2007)), can not be used to address this type of issue directly. In this section, a multistage startup concept will be introduced briefly followed by the detailed problem formulation. A tailored decomposition algorithm is then described.

\subsection{Multistage startup strategy}

The proposed startup policy is schematically illustrated in Fig. 2. The transient startup period is divided into $S$ substages. Over stage $j$ it is assumed that one set of piece-wise constant operating conditions denoted by vector $u_{j}$ is used and $N_{j}$ port switches occur, $S \geq 2, N_{j} \geq 1, j=1,2, \ldots, S$. The primary task is to adjust $u_{j}(j=1,2, \ldots, S)$ in such a way that the process can be driven to track the reference CSS profile $C_{a x}^{*}(t)$ in an optimum fashion while respecting constraints imposed during startup. It is assumed that at the end of stage $S$ the desired profile can be reached. However, since the time required to reach the CSS profile can not be determined a priori, the number of stages $S$ remains unknown.

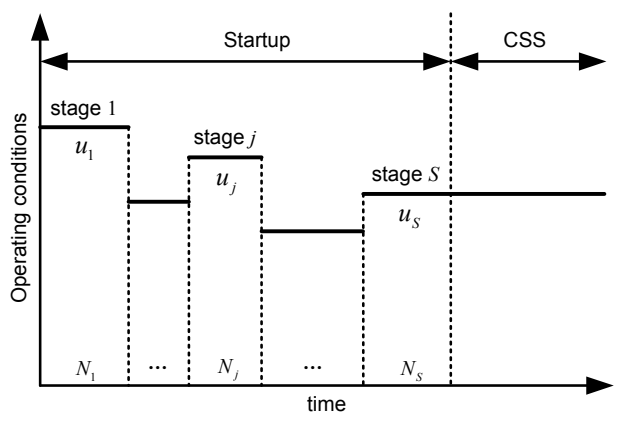

Fig. 2. Illustration of multistage startup strategy for SMB

\subsection{Problem formulation}

In stage $j$, the switching period $t_{S, j}$ and the four dimensionless $m$-factors (Mazzotti et al. (1997)) defined as

$$
m_{i, j}=\frac{Q_{i, j} t_{S, j}-V_{C o l} \epsilon}{V_{C o l}(1-\epsilon)}, i=I, I I, I I I, I V
$$

were chosen as the operating variables and thus $u_{j}=$ $\left[m_{I, j}, m_{I I, j}, m_{I I I, j}, m_{I V, j}, t_{S, j}\right]^{T}, j=1,2, \ldots, S$. In (11), $Q_{i, j}$ is the flow-rate in zone $i$ and $V_{C o l}$ the column volume. Accordingly, the vector of the CSS conditions $u^{*}=\left[m_{I}^{*}, m_{I I}^{*}, m_{I I I}^{*}, m_{I V}^{*}, t_{S}^{*}\right]^{T}$.

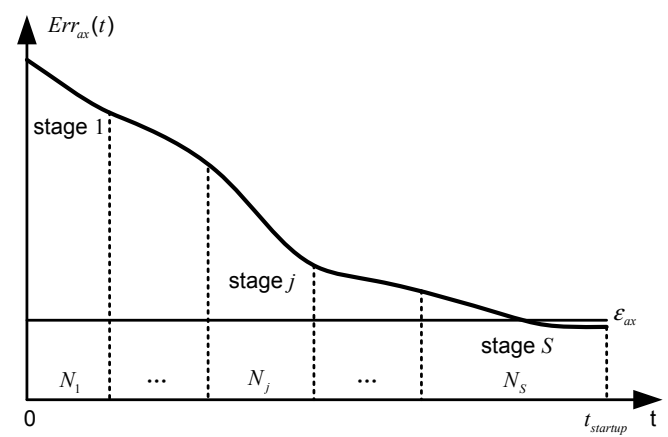

Fig. 3. Development of axial concentration offset during startup

During startup, the deviation of the axial concentration $C_{a x}(t)$ with respect to the nominal one at time $t$ can be characterized in terms of

$$
\operatorname{Err}_{a x}(t)=\left\|C_{a x}(t)-C_{a x}^{*}(t)\right\|_{2}^{2}, t \geq 0
$$

Note that both $C_{a x}(t)$ and $C_{a x}^{*}(t)$ in (12) are discretized state vectors. With an appropriate startup policy, the offset value decreases as the axial concentration gradually approaches the reference profile. The development of $\operatorname{Err}_{a x}(t)$ is exemplarily shown in Fig. 3. Suppose that at the end of stage $S$, the deviation fulfills the specified tolerance $\epsilon_{a x}$. The total number of cycles required is then denoted as $N_{C S S}$ and the corresponding time is defined as the startup time $t_{\text {startup }}$.

A straightforward aim of a startup optimization problem

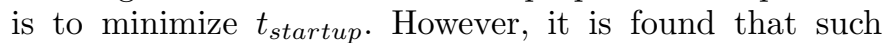
selection of the cost function leads to ill-conditioning for our problem. Alternatively, we employ a different objective function in this study. A time integration of (12) over startup is introduced:

$$
J=\int_{0}^{t_{\text {startup }}} \operatorname{Err}_{a x}(t) d t
$$

Obviously, one set of operating conditions which enables a smaller value of $J$ imply a relatively shorter transient time and lower overall solvent usage, and thus is more preferable than others. Therefore, it is reasonable to use the performance criterion $J$ to quantitatively characterize the startup behavior. A similar objective function was also used by Wozny and Li (2004) for the startup optimization of distillation columns. The multistage optimal startup problem is formulated as below:

$$
\min _{u} J
$$

s.t.

$$
\begin{gathered}
\left.\operatorname{Err}_{a x}(t)\right|_{t=t_{\text {startup }}} \leq \epsilon_{a x} \\
Q_{I, j} \leq Q_{\max }, Q_{I I I, j} \leq Q_{\max } \\
m_{i, S}=m_{i}^{*}, t_{S, S}=t_{S}^{*} \\
m_{I, j}-m_{I I, j}>0, m_{I, j}-m_{I V, j}>0 \\
m_{I I I, j}-m_{I I, j}>0, m_{I I I, j}-m_{I V, j}>0
\end{gathered}
$$


where $u=\left[u_{1}^{T}, u_{2}^{T}, \ldots, u_{S}^{T}\right]^{T}$, is the vector of the startup operating conditions. $Q_{\max }$ represents the maximum allowable flow-rate in zones $I$ and $I I I$. One set of equality constraints considered in (14) enforces the process to use the nominal CSS conditions $u^{*}$ at stage $S$.

It is worth nothing that although the nominal concentration profile is assumed to be known, evaluating the objective function exactly with (13) is non-trivial since it requires the knowledge of the profile at every time $t$, $t \in\left[0, t_{\text {startup }}\right]$. Alternatively, the extract integration can be approximated cycle-wise

$$
J \approx \sum_{j=1}^{S} \tilde{J}_{j}=\sum_{j=1}^{S} \sum_{k=1}^{N_{j}} \operatorname{Err}_{a x, j}^{k} t_{S, j}
$$

where $\tilde{J}_{j}$ represents the approximated value for the integration over stage $j, \operatorname{Err}_{a x, j}^{k}$ the offset value at the end of cycle $k$ of the same stage. With the approximation approach, the desired CSS concentration profile only at the end of one cycle is required, which allows a significantly easier numerical implementation.

\subsection{Decomposition strategy}

It should be pointed about that in the above formulation, the stage interval $N_{j}(j=1,2, \ldots, S)$ are assumed to be fixed to reduce complexity, even if they are also degrees of freedom. However, we found that problem (14) still remains intractable. This motivates us to propose a sequential decomposition algorithm to guarantee numerical tractability. The strategy decomposes the original problem into a sequence of subproblems. In particular, for stage $j$ the corresponding problem is stated as follows:

$$
\operatorname{Prob}_{j}=\min _{u_{j}}\left(\tilde{J}_{j}+\epsilon_{r e g}\left\|u_{j}-u^{*}\right\|_{2}^{2}\right)
$$

s.t.

$$
\begin{gathered}
Q_{I, j} \leq Q_{\max }, Q_{I I I, j} \leq Q_{\max } \\
m_{I, j}-m_{I I, j}>0, m_{I, j}-m_{I V, j}>0 \\
m_{I I I, j}-m_{I I, j}>0, m_{I I I, j}-m_{I V, j}>0
\end{gathered}
$$

Note that the equality constraints imposed on the operating conditions of stage $S$ in (14) can not be explicitly treated in this strategy. Alternatively, an additional regularizing term with the coefficient $\epsilon_{r e g}$ is added to the objective function to guide the solution towards the CSS operating point as the subproblems are solved stage by stage.

To solve $\operatorname{Prob}_{j}$ the concentration profile at the end of stage $j-1$ with the optimum operating point $u_{j-1}^{*}$ i.e. $C_{a x, j-1}^{f i n a l, *}$, is required to initialize the concentration profile at the beginning of stage $j C_{a x, j}^{i n i t}$. As assumed in section 2 , initially the columns are empty and thus $C_{a x, 0}^{f i n a l, *}$ is specified by a zero vector. The stage-wise subproblems are then solved in sequence until at the end of stage $S$ the corresponding tracking error $\operatorname{Err}_{a x, S}$ fulfills the required tolerance $\epsilon_{a x}$. The decomposition algorithm described above is outlined in Fig. 4. It should be stressed that the solution obtained by the algorithm is only an approximation to that of the original problem (14). We do not pursue any further refinement of the solution in this paper.

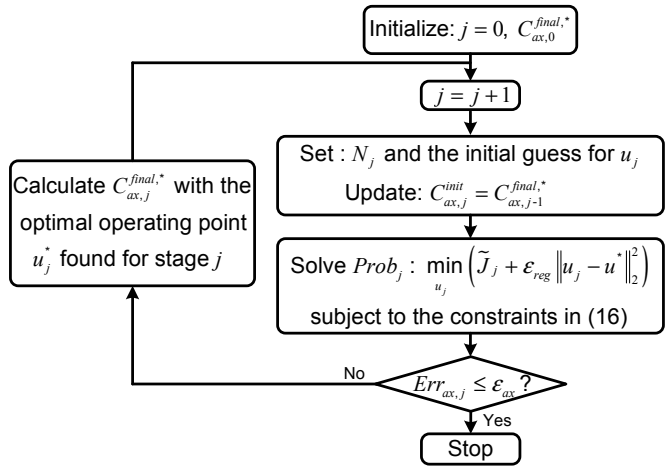

Fig. 4. Decomposition algorithm developed for solving multistage startup of SMB

\section{RESULTS AND DISCUSSION}

\subsection{Example process}

A laboratory-scale binary separation was chosen as an illustrating example to study the new startup strategy. The detailed parameters used to quantify the model process are summarized in Table 1. The nonlinear isotherm coefficients are based on the experimental data which describe the separation of cycloheptanone and cyclohexanone on silica using n-hexane:ethylacetate (85:15) as mobile phase. For the linear case, the parameters $K_{i}(i=A, B)$ were set to zero. The feed concentration of both components was fixed at $1 \mathrm{~g} / \mathrm{L}$, and the maximum allowable flow-rate $Q_{\max }$ was restricted to $10 \mathrm{~mL} / \mathrm{min}$. For OCFE discretization, the number of finite elements $\left(N_{F e}\right)$ and internal collocation points $\left(N_{C p}\right)$ used for each column is also given in Table 1. All the following case studies were performed on a Pentium D 3.0 GHz Linux machine with 2 Gb RAM memory.

Table 1. Parameters for the example process

\begin{tabular}{cccc}
\hline \multicolumn{4}{c}{ Column properties and operating parameters: } \\
Configuration & $1-1-1-1$ & $C_{i}^{F}, i=A, B(\mathrm{~g} / \mathrm{L})$ & 1 \\
Dimensions $(\mathrm{cm})$ & $1 \times 10$ & $Q_{\max }(\mathrm{mL} / \mathrm{min})$ & 10 \\
$\epsilon(-)$ & \multicolumn{4}{c}{0.667} & $N T P$ per column $(-)$ & 40 \\
& Adsorption isotherm coefficients: \\
$H_{A}(-)$ & 5.078 & $K_{A}(\mathrm{~L} / \mathrm{g})$ & 0.089 \\
$H_{B}(-)$ & 5.718 & $K_{B}(\mathrm{~L} / \mathrm{g})$ & 0.105 \\
& Discretization & \\
$N_{F e}$ & 5 & $N_{C p}$ & 3 \\
\hline
\end{tabular}

\subsection{CSS optimization}

The CSS operating point $u^{*}$ was obtained from solving the following feed throughput maximization problem for the example described in section 5.1:

$$
\max _{u} Q_{F}
$$

s.t.

$$
\begin{gathered}
\left\|C_{a x}^{k+1}-C_{a x}^{k}\right\|_{2} \leq \epsilon_{\text {steady }} \\
\operatorname{Pur}_{E x} \geq \operatorname{Pur}_{E x, \min } \\
\operatorname{Pur}_{R a f} \geq \operatorname{Pur}_{R a f, \min } \\
Q_{I} \leq Q_{\max }
\end{gathered}
$$

where $C_{a x}^{k}$ and $C_{a x}^{k+1}$ represent the axial concentration profiles at the end of cycle $k$ and $k+1$, the vector of decision variables $u=\left[m_{I}, m_{I I}, m_{I I I}, m_{I V}, t_{S}\right]^{T}, \epsilon_{\text {steady }}$ the 
CSS tolerance, Pur Ex,min and Pur Raf,min the minimum purity requirements for the extract and raffinate products, respectively

The single-discretization approach equipped with a sequential quadratic programming (SQP) solver (E04UCF from the NAG library) was adopted to solve the CSS optimization problem (17). Both Pur Ex,min $_{\text {and }}$ Pur $_{R a f, \min }$ were specified as $90 \%$. The normalized concentration profiles with respect to the feed concentrations were used to check CSS numerically and $\epsilon_{\text {steady }}=1.0 \times 10^{-4}$. The gradients of the purity constraints which can not evaluated analytically were approximated by a finite difference method. The optimum operating conditions are reported in Table 2. Following the conventional startup policy, the desired CSS profile can be attained after 107 and 115 cycles for the linear and nonlinear case, respectively, and the startup time for both cases is more than 3.8 hours.

Table 2. Optimized CSS operating points and performance of conventional startup

\begin{tabular}{ccc}
\hline & Linear case & Nonlinear case \\
\hline$m_{I}^{*}$ & 6.2337 & 6.1712 \\
$m_{I I}^{*}$ & 5.3241 & 5.1839 \\
$m_{I I I}^{*}$ & 5.4474 & 5.2818 \\
$m_{I V}^{*}$ & 4.6565 & 4.6034 \\
$t_{S}^{*}(\min )$ & 2.1542 & 2.1379 \\
$N_{C S S}$ & 107 & 115 \\
$t_{\text {startup }}(\mathrm{h})$ & 3.8417 & 4.0976 \\
\hline
\end{tabular}

\subsection{Multistage startup optimization}

The same single-discretization method was also used to sequentially solve the decomposed subproblems. For Prob ${ }_{j}$, $\tilde{J}_{j}$ in the cost function of (16) was determined through the cycle-wise approximation over stage $j$ :

$$
\tilde{J}_{j}=\sum_{k=1}^{N_{j}} \operatorname{Err}_{a x, j}^{k} t_{S, j}
$$

where $\operatorname{Err}_{a x, j}^{k}$ was evaluated with the normalized concentration profiles, and the stage interval $N_{j}$ was fixed at 4 a priori. The optimum solution obtained from stage $j$ was chosen as the initial guess for the subproblem of the successive stage $j+1$. Thus, only the starting point of the optimizing variables for the first stage needs to be specified. In addition, $\epsilon_{a x}=1.0 \times 10^{-6}$ and $\epsilon_{\text {reg }}=1.0 \times$ $10^{-3}$ for the following case studies.

Table 3. Optimal startup operating conditions

\begin{tabular}{|c|c|c|c|c|c|c|}
\hline$j$ & $m_{I, j}^{*}$ & $m_{I I, j}^{*}$ & $m_{I I I, j}^{*}$ & $m_{I V, j}^{*}$ & $t_{S, j}^{*}(\min )$ & $E r r_{a x, j}$ \\
\hline 1 & 5.5066 & 4.7841 & 5.5066 & 5.5066 & 1.9641 & $6.5 \times 10^{-1}$ \\
\hline 2 & 5.4526 & 5.4526 & 5.4526 & 5.4526 & 1.9499 & $5.9 \times 10^{-2}$ \\
\hline 3 & 5.9087 & 5.3839 & 5.4103 & 4.6161 & 2.0692 & $6.7 \times 10^{-3}$ \\
\hline 4 & 6.3054 & 5.3099 & 5.4562 & 4.6835 & 2.1730 & $1.3 \times 10^{-3}$ \\
\hline 5 & 6.1955 & 5.3306 & 5.4434 & 4.6548 & 2.1442 & $2.3 \times 10^{-4}$ \\
\hline 6 & 6.2358 & 5.3234 & 5.4483 & 4.6605 & 2.1548 & $6.6 \times 10^{-5}$ \\
\hline 7 & 6.2290 & 5.3249 & 5.4467 & 4.6567 & 2.1530 & $1.5 \times 10^{-5}$ \\
\hline 8 & 6.2336 & 5.3241 & 5.4473 & 4.6569 & 2.1542 & $4.3 \times 10^{-6}$ \\
\hline 9 & 6.2334 & 5.3242 & 5.4472 & 4.6563 & 2.1541 & $1.1 \times 10^{-6}$ \\
\hline 10 & 6.2340 & 5.3241 & 5.4473 & 4.6562 & 2.1543 & $2.6 \times 10^{-7}$ \\
\hline \multicolumn{2}{|c|}{$N_{C S S}$} & 40 & \multicolumn{3}{|c|}{$t_{\text {startup }}(\mathrm{h})$} & 1.4047 \\
\hline
\end{tabular}

The optimal startup operating conditions and the resulting transient duration are summarized in Table 3 for the linear case. The results for the nonlinear isotherm are quantitatively similar and thus omitted for brevity. The computation time in each case is below 3.5 CPU minutes. With stage increasing, it is found that the tracking error dramatically decreases and the operating point gradually converges to the nominal CSS conditions shown in Table 2. For both cases, totally 10 stages with 4 cycles for each stage are required for the process to reach the reference concentration profile with the given tolerance $1.0 \times 10^{-6}$. Compared to that of the conventional regime, the transient time is reduced by more than $63 \%$ and $65 \%$ for the linear and nonlinear isotherm, respectively. The total amount of desorbent consumed during startup is saved by over $70 \%$.
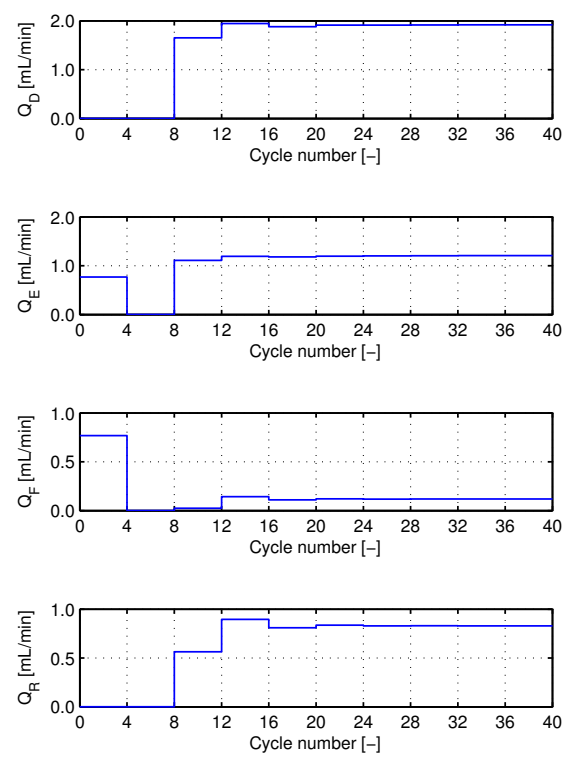

Fig. 5. Transient profile of the external flow-rates during startup for the nonlinear case

The transient profile of the external flow-rates during the startup period is illustrated in Fig. 5 for the nonlinear case. It can be seen that $Q_{D}$ in the first two stages tends to be zero. $Q_{F}$ in the first stage is $0.7674 \mathrm{~mL} / \mathrm{min}$ which is more than 6.4 times high than that at CSS. These results clearly indicate that in order to accelerate the establishment of the desired CSS profile, the feed stream should be "filled into" the columns as much as possible while the desorbent stream has to be "shut off" to avoid dilution. This interesting finding is very similar to the pre-loading concept (Xie et al. (2003)). Furthermore, a decreasing value of $Q_{F}$ in the second stage aims at preventing potential "overload". On the other hand, the development of two outlet flow-rates over the first two stages is also rather enlightening. For the separation under study, it is found that the raffinate stream has began to elute out during the first cycle. However, the elution of the extract is delayed for a few cycles and thus lags behind that of the raffinate. As a result, a value near zero for $Q_{R}$ prevents the raffinate concentration plateau accumulated inside the columns eluting out of the system. A high flow-rate value in the first stage for $Q_{E}$ implies that the process attempts to "throw away" the solvent residing in the columns and reduce dilution, since almost no extract appears at the outlet during this stage; once 
the extract begins to come out, just like $Q_{R}, Q_{E}$ also approaches zero to "shut off" the outlet. The behavior observed is extremely advantageous for building up the CSS profile quickly. Finally, all the parameters shown in Fig. 5 have almost converged towards the CSS operating conditions after about 5 or 6 stages, which reveals that the first several stages play a dominant role in shortening the transient time.

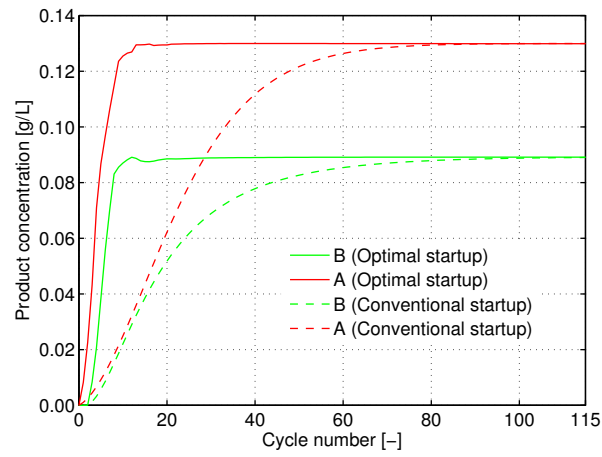

Fig. 6. Comparison of the transient product concentration with different startup strategies (nonlinear isotherms)

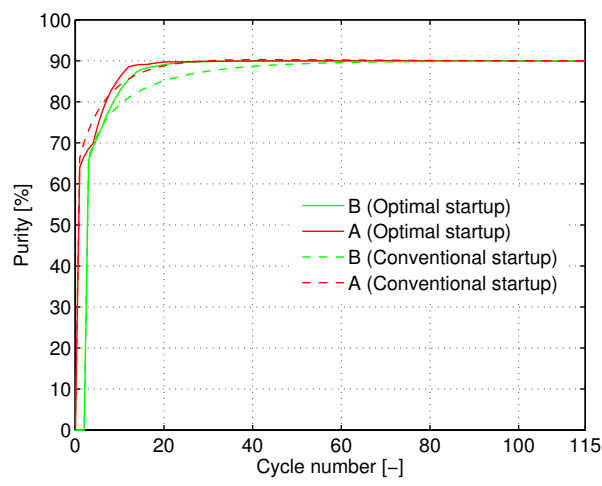

Fig. 7. Comparison of the transient product purity with different startup strategies (nonlinear isotherms)

The startup performance in terms of the product concentration and purity for the nonlinear case is shown in Figs. 6 and 7 , respectively. For the sake of comparison, the results obtained with the conventional startup mode are also given. The process was operated for the same 115 cycles for both startup methods. Note that only after this cycle the nominal CSS profile was found to be established with the conventional scheme. From Fig. 6, it is clearly seen that the optimum startup allows the process to yield more concentrated transient outlet streams. For the example examined here, after only 20 cycles (i.e., 5 stages) both extract and raffinate concentration have almost reached their nominal values. However, more than 80 switching periods are required for the normal startup operation. For the purity, the value of each product is slightly lower than that of the conventional startup in the first two stages. This can be justified by the previous fact that initially the primary task of the process is to load the columns with the feed and not to achieve a good separation. Thereafter, both purity values rapidly converge towards the desired purity of $90 \%$. In contrast, the conventional startup leads to a sluggish purity development, which is particularly obvious for the extract product.

\section{CONCLUSIONS}

In this work a multistage startup strategy has been presented for SMBs. The optimal startup problem has been formulated with the primary purpose of reducing the transient deviation from the desired CSS concentration profile as quickly as possible. To ensure numerical solvability, a specially tailored solution approach has been developed. A laboratory-scale separation has been used to illustrate the feasibility of the decomposition algorithm and quantitatively assess the performance of the new startup regime. It has been found that with the aid of the optimal startup operation, the process under examination achieves over $60 \%$ reduction in startup time and more than $70 \%$ savings in desorbent consumption. Moreover, the transient performance in terms of product concentration and purity is considerably enhanced when compared to that of the conventional startup.

As pointed out, the stage length is specified a priori in this work. The proposed decomposition approach can guarantee only the stage-wise optimality of solution. The solution obtained may be suboptimal for the original problem. Future work will examine the effect of the stage length on the transient behavior, implement an adaptive length strategy, and explore alternative problem formulations and solution approaches. Experimental validation of the optimal startup operation is another interesting subject.

\section{REFERENCES}

Dünnebier, G., Fricke, J., and Klatt, K.U. (2000). Optimal design and operation of simulated moving bed chromatographic reactors. Ind. Eng. Chem. Res., 39, 2290-2304.

Guiochon, G., Felinger, A., Shirazi, D., and Katti, A. (2006). Fundamentals of preparative and nonlinear chromatography. Academic Press, Elsevier, Amsterdam.

Kawajiri, Y. and Biegler, L.T. (2006). Large scale nonlinear optimization for asymmetric operation and design of simulated moving beds. J. Chromatogr. A, 1133, 226240.

Li, S. and Petzold, L. (1999). Design of new DASPK for sensitivity analysis. Technical Report TRCS99-28, University of California, Santa Barbara, CA, USA.

Lim, B.G. and Ching, C.B. (1996). Modelling studies on the transient and steady state behaviour of a simulated counter-current chromatographic system. Sep. Technol., $6,29-41$.

Ma, Z. and Wang, N.H.L. (1997). Standing wave analysis of SMB chromatography: linear systems. AIChE J., 43, 2488-2508.

Mazzotti, M., Storti, G., and Morbidelli, M. (1997). Optimal operation of simulated moving bed units for nonlinear chromatographic separations. J. Chromatogr. A, $769,3-24$.

Toumi, A., Engell, S., Diehl, M., Bock, H., and Schlöder, J. (2007). Efficient optimization of simulated moving bed processes. Chem. Eng. Process., 46, 1067-1084.

Wozny, G. and Li, P. (2004). Optimisation and experimental verification of startup policies for distillation columns. Comput. Chem. Eng., 28, 253-265.

Xie, Y., Mun, S.Y., and Wang, N.H.L. (2003). Startup and shutdown strategies of simulated moving bed for insulin purification. Ind. Eng. Chem. Res., 42, 1414-1425. 